we found no relationship between oestrogen receptor status and lymph node metastases, so these two independent prognostic factors may be used together to provide a more accurate prediction of prognosis. This may be of particular value in planning the entry criteria and stratification of future adjuvant trials.

Clearly, patients with tumours that contain oestrogen receptors but who have no axillary metastases have a good prognosis, and adjuvant treatment would seem inappropriate for this subgroup. Conversely, patients with node metastases and tumours without receptors have a poor prognosis, the worst of all the subgroups studied.

Patients without oestrogen receptors and with no axillary metastases have been identified as a high-risk group, and it would now seem appropriate to include such patients in future trials of adjuvant therapy in early breast cancer.

We thank the many surgeons on Merseyside who co-operated in this study. The tumours were collected by Mrs Patricia Clark, who also gave invaluable secretarial help. This investigation was supported by a grant from the Mersey Regional Health Authority.

\section{References}

${ }^{1}$ McGuire WL, Carbone PP, Volmer EP, eds. Estrogen receptors in human breast cancer. New York: Raven Press, 1975.

${ }^{2}$ Knight WA, Livingston RB, Gregory EJ, McGuire WL. Estrogen receptor as an independent prognostic factor for early recurrence in breast cancer. Cancer Res $1977 ; 37: 4669-71$.

${ }^{3}$ Maynard PV, Blamey RW, Elston CW, Haybittle JL, Griffiths K. Estrogen receptor assay in primary breast cancer and early recurrence of the disease. Cancer Res 1978;38:4292-5.

${ }^{4}$ Rich MA, Furmanski P, Brooks SC. Prognostic value of Estrogen Receptor determinations in patients with breast cancer. Cancer Res 1978;38: 4296-8.

${ }^{5}$ Cooke T, George D, Shields R, Maynard P, Griffiths K. Oestrogen receptors and prognosis in early breast cancer. Lancet 1979 ;i:995-7.

${ }^{6}$ Bishop HM, Blamey RW, Elston CW, Haybittle JL, Nicholson RI, Griffiths K. Relationship of oestrogen receptor status to survival in breast cancer. Lancet 1979;ii:283-4.

${ }^{7}$ Peto R, Pike MC, Armitage NE, et al. Design and analysis of randomised clinical trials requiring prolonged observation of each patient. II. Analysis and Examples. Br $\mathcal{F}$ Cancer 1977;35:1-39.

(Accepted 12 August 1981)

\title{
Flexible sigmoidoscopy in outpatients with suspected colonic disease
}

\author{
C O RECORD, M G BRAMBLE, A H LISHMAN, G I SANDLE
}

\begin{abstract}
One hundred and fifteen patients attending a gastroenterology clinic were investigated by flexible sigmoidoscopy as outpatients. They were asked to fast before the examination and given $a$ high-volume enema and sedated before the examination. A standard long colonoscope was used rather than the $60-\mathrm{cm}$ sigmoidoscope, which limits the distance that can be examined and forces the operator to work very close to the patient. Preparation was considered good in 95 patients and 49 were examined as far as the hepatic flexure or beyond. Sixty-one patients were found to have lesions of the colon, 25 of them ulcerative colitis, 16 a polyp, and nine carcinoma.

Despite the fact that these patients were selected (some of them had already had ulcerative colitis diagnosed), flexible sigmoidoscopy proved to be a valuable initial outpatient investigation. The proximal colon was well visualised in 46 patients and a subsequent barium enema was considered unnecessary. There were no complications and the procedure seemed to be well tolerated.
\end{abstract}

\section{Introduction}

Colonoscopy has become an accepted method of investigating the colon, but, unlike upper gastrointestinal endoscopy, the procedure is not widely practised in the United Kingdom outside

\footnotetext{
Gastroenterology Unit, Royal Victoria Infirmary, Newcastle upon Tyne

C O RECORD, DPHIL, FRCP, consultant physician in gastroenterology $M$ G BRAMBLE, MRCP, senior registrar

A H LISHMAN, MRCP, first assistant

G I SANDLE, MD, first assistant
}

specialist centres and many instruments are underused. This is partly because colonoscopy is usually regarded as difficult and partly because elaborate bowel cleaning techniques are frequently advocated. Recently $60-\mathrm{cm}$ flexible sigmoidoscopes have been introduced, and initial studies have suggested that flexible sigmoidoscopy is a greatly superior investigation to rigid sigmoidoscopy and that the diagnostic yield of lesions found is about three times greater with the flexible instrument. ${ }^{2}$ During the past year we have undertaken 115 outpatient sigmoidoscopies using a flexible instrument and we report here our experience with this technique.

\section{Patients and methods}

All 115 patients were attending a gastroenterology outpatient clinic for the diagnosis or treatment of bowel disease. If patients were thought to require sigmoidoscopy they were asked to attend the gastroenterology unit after a period of fasting, where they received a single high-volume enema, consisting of three pints of tap water and $\mathbf{3} \mathbf{g}$ Veripaque (Winthrop; contains $50 \mathrm{mg}$ oxyphenisatin). The enema was given slowly and the patient retained this if possible for 15 minutes. The examination was then carried out after sedation with pentazocine and diazepam. One to two hours after the procedure the patients were allowed home, accompanied by a relative or friend. No dietary manipulations or laxatives were prescribed in the 24 hours before the examination.

We chose to use standard long colonoscopes for the procedure rather than be limited to $60 \mathrm{~cm}$ and be working very close to the patient, as is necessary with the flexible sigmoidoscope.

\section{Results}

Preparation was deemed to be good in $95(82 \%)$ of patients, and in $49(43 \%)$ it allowed examination of the colon to the hepatic flexure or beyond. In 37 the examination was terminated after inspection of the rectum and sigmoid colon only.

In $61(53 \%)$ patients a lesion in the colon was found, the principal diagnosis being ulcerative colitis ( 25 patients), while polyps (16) were 
the next most common lesion. In two patients histological examination showed that the polyps were malignant and a further nine patients had a carcinoma. The remaining patients had benign strictures ( 5 cases), diverticular disease (3), and miscellaneous diagnoses (3).

\section{Discussion}

Flexible sigmoidoscopy is a valuable initial investigation in patients with colonic symptoms and can be carried out on outpatients in a similar way to that practised widely for the examination of the upper gastrointestinal tract. Elaborate home or hospital preparation is not necessary, $82 \%$ of patients being well prepared after a single high-volume enema. In $53 \%$ of patients a lesion was found at the time of the examination. The series consisted, however, of selected patients, in some of whom a diagnosis of ulcerative colitis had already been established but the examination had been deemed necessary as part of their management. In 46 patients the proximal colon was easily visualised at this first examination and in this group subsequent barium enema was considered unnecessary.

An exact record of the time taken for each examination was not kept and will obviously vary among operators. Nevertheless, the procedure was thought to be comparable to upper gastrointestinal endoscopy and was usually terminated after 10-20 minutes unless a polyp or other lesion was found. We have always used premedication before flexible sigmoidoscopy and agree with Thompson et $a l^{3}$ that endoscopy under sedation is much less unpleasant for the patient.

Two previous studies of flexible sigmoidoscopy ${ }^{2}$ have used a $60-\mathrm{cm}$ instrument and have found lesions in $39 \%$ and $44 \%$ of patients. In these studies low-volume enemas were used in patients who were not sedated. In our study we used the standard long colonoscope for the examination and this allowed the colon beyond the sigmoid to be visualised in $78(68 \%)$ patients. In these patients little additional time was spent on the examination and a greater diagnostic yield was obtained. Short instruments offer little advantage over long instruments, which allow the operator greater freedom of movement at a more comfortable distance from the patient. We still use rigid sigmoidoscopy in some patients, particularly those young patients thought to be suffering from the irritable bowel syndrome on whom complete investigations have not been undertaken and patients with severe symptoms suggestive of ulcerative colitis on whom an immediate sigmoidoscopy is thought to be necessary. There were no complications from flexible sigmoidoscopy, which appears to be a valuable, well tolerated, and simple outpatient procedure for the management of suspected colonic disease.

\section{References}

${ }^{1}$ Bohlman TW, Katon RM, Lipshutz GR, McCool MF, Smith FW Melnyk CS. Fiberoptic pansigmoidoscopy : an evaluation and comparison with rigid sigmoidoscopy. Gastroenterology 1977;72:644-9.

2 Marks G, Whitney-Boggs H, Castro AF, Gathright JB, Ray JE, Salvati E. Sigmoidoscopic examinations with the rigid and flexible fiberoptic sigmoidoscopes in the surgeon's office : a comparative study of effectiveness in 1012 cases. Dis Colon Rectum 1979;22:162-8.

3 Thompson DG, Evans SJ, Murray RS, Lennard-Jones JE, Cowan RE, Wright JT. Patients appreciate premedication for endoscopy. Lancet $1980 ;$ ii :469-70.

(Accepted 12 August 1981)

\title{
Would a medium-nicotine, low-tar cigarette be less hazardous to health?
}

\author{
R STEPNEY
}

\begin{abstract}
Smoking behaviour and exposure to carbon monoxide, nicotine, and tar were studied in 19 middle-tar smokers. All smoked their own brands for three weeks and then switched to either a conventional low-nicotine, low-tar brand (control) or a medium-nicotine, low-tar cigarette for a further three weeks, the order then being reversed. The medium-nicotine, low-tar brand also had a low delivery of carbon monoxide.

With the medium-nicotine, low-tar cigarette mouthlevel delivery and intake of nicotine was similar to that with the smokers' usual brands, and significantly greater than with the control low-tar cigarette. Intake of carbon monoxide from the medium-nicotine, low-tar cigarette was significantly less than with either own or control brands. With both low-tar brands mouth-level exposure to tar was reduced relative to smokers' usual cigarettes. There was no evidence, however, that the reduction in tar exposure was greater with the medium-nicotine
\end{abstract}

University of Cambridge Clinical School, Department of Medicine, Addenbrooke's Hospital, Cambridge CB2 2QQ

R STEPNEY, MA, MSC, research psychologist brand than with the control low-tar cigarette. Both lowtar brands were "oversmoked" relative to subjects' usual middle-tar cigarettes.

The medium-nicotine, low-tar cigarette was marginally more acceptable than the control brand, and the particular design used in the study resulted in a lower intake of carbon monoxide. In terms of reducing mouthlevel exposure to tar, however, the medium-nicotine, low-tar cigarette had no advantage over the control lowtar product. In part this was because the ratio of tar to nicotine delivery obtained by human smokers was not the same as that obtained by smoking machine.

\section{Introduction}

Smokers unwilling to abstain have been encouraged to smoke cigarettes with low yields of tar. ${ }^{12}$ Though almost all smokers have tried low-tar brands, however, only a few have switched permanently to them. (In 1977 only $12 \%$ of cigarettes sold in Britain were in the Government's low-tar category. ${ }^{3}$ ) Moreover, those smokers who have switched smoke more intensively, taking a greater volume of smoke (personal paper presented to a meeting on carbon monoxide and smoke intake, Southampton, March 1980) and inhaling more deeply. ${ }^{4}$ Any reduction in actual tar exposure is therefore likely to be considerably smaller than 